\title{
Negation in Cognitive Reasoning ${ }^{\star}$
}

\author{
Claudia Schon ${ }^{1[0000-0003-2455-0974]}$, Sophie Siebert ${ }^{2}[0000-0002-6812-796 X]$, and Frieder \\ Stolzenburg 2[0000-0002-4037-2445] \\ 1 Universität Koblenz-Landau, Institute for Web Science and Technologies, Universitätsstr. 1, \\ 56070 Koblenz, Germany, schon@uni-koblenz.de, http://www.uni-koblenz.de/ \\ 2 Harz University of Applied Sciences, Automation and Computer Sciences Department, \\ Friedrichstr. 57-59, 38855 Wernigerode, Germany, \\ \{ssiebert,fstolzenburg\}@hs-harz.de, http://artint.hs-harz.de/
}

\begin{abstract}
Negation is both an operation in formal logic and in natural language by which a proposition is replaced by one stating the opposite, as by the addition of "not" or another negation cue. Treating negation in an adequate way is required for cognitive reasoning, which aims at modeling the human ability to draw meaningful conclusions despite incomplete and inconsistent knowledge. One task of cognitive reasoning is answering questions given by sentences in natural language. There are tools based on discourse representation theory to convert sentences automatically into a formal logic representation, and additional knowledge can be added using the predicate names in the formula and knowledge databases. However, the knowledge in logic databases in practice always is incomplete. Hence, forward reasoning of automated reasoning systems alone does not suffice to derive answers to questions because, instead of complete proofs, often only partial positive knowledge can be derived, while negative knowledge is used only during the reasoning process. In consequence, we aim at eliminating syntactic negation, strictly speaking, the negated event or property. In this paper, we describe an effective procedure to determine the negated event or property in order to replace it by its inverse. This lays the basis of cognitive reasoning, employing both logic and machine learning for general question answering. We evaluate our procedure by several benchmarks and demonstrate its practical usefulness in our cognitive reasoning system.
\end{abstract}

Keywords: cognitive reasoning $\cdot$ negation $\cdot$ automated reasoning.

\section{Introduction}

Negation is a very common operation in natural language. It reverses the meaning of a statement or parts of it. Especially in text comprehension, it is important to correctly identify and process negation because negation can strongly alter the meaning of the overall contents of a statement. In this paper, we focus on solving commonsense reasoning tasks in natural language, which requires an adequate handling of negation.

Commonsense reasoning is the sort of everyday reasoning humans typically perform about the world [31]. It needs a vast amount of implicit background knowledge,

\footnotetext{
* The authors gratefully acknowledge the support of the German Research Foundation (DFG) under the grants SCHO 1789/1-1 and STO 421/8-1 CoRg - Cognitive Reasoning.
} 
such as facts about objects, events, space, time, and mental states. While humans solve commonsense reasoning tasks relatively easily and intuitively, they are rather difficult for a computer.

Most approaches that tackle commonsense reasoning and its benchmarks do not use any reasoning at all and rely mainly on machine learning and statistics on natural language texts. These often very successful approaches combine unsupervised pre-training on very large text corpora with supervised learning procedures $[13,34]$. However, the good results currently achieved, e.g., with BERT [13] should be critically questioned. [32] presents a system based on BERT that is only slightly below the average untrained human baseline on the Argument Reasoning Comprehension Task [15], but a close examination of the learned model by the authors of [32] shows that there are statistical cues in the dataset that have nothing to do with the represented problems. The good performance of BERT is explained by BERT exploiting these cues. Examples of those cues are the words "not", "is", "do", "are", "will not" and "cannot", with "not" being the strongest cue. After creating a version of the dataset without the bias of the cue "not", the authors find that performance of BERT drops to the random baseline. This illustrates that the system based on BERT in [32] has no underlying understanding of the tasks. This observation emphasizes the importance of explanations in commonsense reasoning and negation handling.

Therefore, we take a different way and rely on background knowledge represented in logic together with automated reasoning (cf. [36]). In a first step, the natural language representation of a benchmark problem is translated into a first-order logic formula using the KnEWS system [7] - a tool based on discourse representation theory [20]. Since many of the benchmark problems contain negations, it is important that they are treated correctly. Taking a look back at the detected bias from [32], it becomes clear that negation in particular is responsible for the majority of the biases. The word "not" is not only the strongest cue, but other cues are compound of it. This requires increased caution when dealing with negations.

By eliminating the word "not", additional technical advantages apply: While KnEWS can successfully detect negation, it often generates formulae with a large negation scope. Often it spans a whole sentence or subphrase. Our goal is to localize the negation as precisely as possible and thus to pinpoint the exact negated event or property, which can later be replaced by its non-negative counterpart or antonym - the inverse of the negated event or property. In many cases, the negation can even be completely removed from the formula in this way, which often enables an automated reasoning system to derive more positive knowledge. In the CoRg system [35] (cf. Section 3.1), we use this knowledge as input for a neural network calculating a likelihood for each answer alternative to fit to the respective premise. A precisely determined (scope of the) negation in the formal representation also erases ambiguity and facilitates further processing.

In this paper, we therefore present an approach to automatically identify a negated event or property using a natural language sentence and the corresponding logical formula generated by KnEWS. Section 2 describes negation in logic as well as natural language and its importance in cognitive reasoning, which aims at modeling the human ability to draw meaningful conclusions despite incomplete and inconsistent knowledge 
[14]. In Section 3, we describe the cognitive reasoning system CoRg, in which this work is embedded, including challenges caused by negation. In Section 4, we present experimental results and demonstrate the usefulness of our approach for cognitive reasoning. Section 5 contains a summary, conclusions, and an outlook for future work.

\section{Background and Related Works}

\subsection{Negation in Logic and Natural Language}

Negation is a complex phenomenon in both logics and natural language [16]. In formal logics, the meaning of negation seems to be easy: It is a unary operation toggling the truth value of statements between true and false. In most classical logics, we have that double negation does not alter the original truth value, whereas this might not be true in other logics, e.g., multi-valued logics. In any case, the scope of a negation is the subformula that is affected by the negation. There are several types of negation in special logics: For instance, negation as failure or default negation is a non-monotonic inference rule in logic programming, used to derive not $p$ for some statement $p$ from the failure to derive $p$ provided that this is consistent with the current knowledge [4]. In argumentation logics, strict and defeasible negation is distinguished $[37,39]$. The latter may be debated in the course of an argumentation process until an argument is defeated or ultimately justified.

In natural language, the meaning of negation often is not that clear, and it consists of several components (cf. [18]): Instead of precisely defined negation operators, there is a negation cue, that may be a syntactically independent negation marker like "never", "nor", "not" (syntactic negation), an affix expressing negation like "i(n)-" and "un-" (morphological negation), or a word whose meaning has a negative component like "deny" (lexical negation). The scope of a negation is the whole part of the sentence or utterance that is negated, i.e., affected by the negation cue.

Often the effect of negation shall be localized more precisely and reduced to only one part of the scope. On the one hand, there is the negated event or property, which we call negatus henceforth, usually a verb, noun, or adjective, that is directly negated. On the other hand, the focus is the part of the scope that is most prominently negated. It can also be defined as the part of the scope that is intended to be interpreted as false or whose intensity is modified [18]. By this, eventually a positive meaning may be implied. For example, the sentence "They didn't release the UFO files until 2008." has the negation cue "-n't". The pragmatic meaning of the sentence is probably a positive one, namely "The UFO files were released in 2008.", since "until 2008" is the negation focus [29]. Nevertheless, the negatus is "release" inducing the temporal negative meaning of the sentence: "They did not release the files before 2008 ".

\subsection{Commonsense Reasoning and Negation}

For automated commonsense reasoning, negation in natural language has to be treated in a formal manner. Traditional approaches tackle this problem by using Montague semantics together with Kripke semantics for modal logics [17]. Here, negation often is 
discussed in the context of performative verbs, e.g., "promise". So the two negations in "I do not promise not to assassinate the prime minister." [25] refer to different parts of the sentence and thus do not annihilate each other like double negation in classical logics. A more recent approach for formal negation detection comes from discourse representation theory and categorial grammars [20]. By means of the NLP toolchain [6], negation is detected on the basis of this theory. By means of further tools like KnEWS [7], complete sentences possibly including negation can be transformed eventually into a first-order logic formalization. Nevertheless, all these procedures and tools cannot hide the fact that the meaning of negation in natural language may be highly ambiguous. In languages like French or German, e.g., even the scope of negation may not be clear because of syntactic ambiguity. The German utterance "Ich verspreche dir nicht ruhig zu bleiben." may mean "I do not promise to stay calm." or "I promise not to stay calm."

As written in the introduction, the motivation to investigate negation is to treat it adequately in the context of cognitive reasoning. For this, it is important to identify a negation by its cue and assign the corresponding negatus correctly. For evaluation of our approach, there are numerous benchmark sets for commonsense reasoning and negation: The COPA Challenge (Choice of Plausible Alternatives) [26] or the StoryClozeTest [30] require causal reasoning in everyday situations, both without special focus on negation, however. In [29], ConanDoyle-neg, a corpus of stories by Conan Doyle annotated with negation information is presented. Here, the negation cues and their scope as well as the negatus have been annotated by two annotators in the CD-SCO dataset. The SFU Opinion and Comments Corpus [21] is a corpus for the analysis of online news comments. One of its focuses is how negation affects the interpretation of evaluative meaning in discourse.

\section{Methods}

\subsection{A System for Cognitive Reasoning}

The project CoRg (Cognitive Reasoning) [35] aims at solving commonsense reasoning benchmark tasks using extensive knowledge bases, automated reasoning, and neural networks. Benchmarks for commonsense reasoning often are multiple-choice natural language question-answering tasks. There, a premise together with answer alternatives is given, and the objective is to find the right alternative. An example for such a task is given in Figure 1. It is taken from the StoryClozeTest benchmark [30], which contains 3,744 problems in total.

Premise: I think yesterday was when I realized I shouldn't be a mathematician. I was at the grocery store, restocking for next week. I had bought around twenty items, and thought I was under my budget. When I went to check out, I was ten dollars over!

Alternative 1: I then realized that I was not good at math.

Alternative 2: Then I knew that I was great at math!

Figure 1. A StoryClozeTest example. 
Most systems tackling this type of problems rely on pre-trained neural networks and fine-tune their network for their specific problem. The natural language input is mapped to word embedding vectors (cf. [19]) and used as input for the neural network. In our approach, however, we enrich the input by knowledge derived through automated reasoning. The whole process is shown in Figure 2: First, we translate the natural language input into a logical formula using KnEWS [7]. This is done for the premise as well as the answer candidates. Thus, we generate three logical formulae.

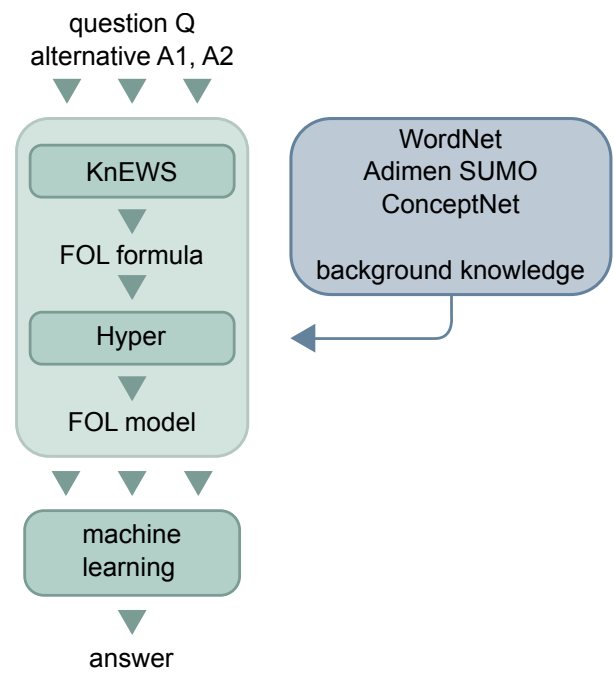

Figure 2. The CoRg system.

In these formulae, the predicate names often correspond to words from the original sentence. Thus, the predicate names are used to look up synonyms from WordNet [27] and logically formalized background knowledge from different ontologies like AdimenSumo [3] and knowledge graphs like ConceptNet [24]. For each of the three formulae generated by KnEWS, the gathered knowledge together with the formula is used as input for the tableaux-based automated reasoning system Hyper [9] to perform inferences. In this process, we do not expect Hyper to find a proof, but use it as an inference engine to derive new knowledge. Hyper thus computes a model or is terminated after a certain time and returns a tableau branch computed up to that point. Since there is the possibility that Hyper would still have closed this branch if it had run longer, we refer to this branch as a potential partial model. The information in the (potential partial) models represents knowledge that can be (potentially) deduced from the original sentence and the gathered background knowledge and thus provides more information than the original natural language sentence alone.

We extract all predicate names from the output of Hyper and use them as input for a two-channel neural network, pairing each answer candidate with the premise. Each word is mapped to a word embedding vector. Here we make use of the 300-dimensional 
word embeddings from ConceptNet Numberbatch [38] which has 400,000 entries. The neural network calculates a likelihood how much the pairs (consisting of question and answer candidate) correlate. Eventually, the answer with the highest likelihood is chosen as the correct answer. For further details about the system, the reader is referred to $[35,36]$.

\subsection{Negation Scope and the Negatus - Why Size Matters}

An unnecessarily large scope of a negation in a formula induces a problem for the CoRg system. To see this, consider the following example (cf. first answer alternative in Figure 1):

"I then realized that I was not good at math."

KnEWS translates this sentence into the following formula:

$$
\begin{aligned}
\exists A, B, C, D, E & (\text { person }(A) \wedge \text { person }(B) \wedge \text { then }(C) \wedge \text { manner }(D, C) \wedge \\
& \text { topic }(D, E) \wedge \operatorname{actor}(D, B) \wedge \operatorname{realize}(D) \wedge \\
& \neg \exists F, G(\operatorname{at}(G, F) \wedge \operatorname{math}(F) \wedge \operatorname{theme}(G, A) \wedge \operatorname{good}(G)))
\end{aligned}
$$

We note that Formula (1), generated by KnEWS, is far from complete and how humans would formalize this sentence. For instance, the generated formula introduces two variables $A$ and $B$, which refer to the two occurrences of the personal pronoun "I", but sound anaphora resolution would yield $A=B$. Furthermore, there is the singleton variable $E$, which actually refers to the subclause "that I was not good at math", but this is not formalized. Nevertheless, Formula (1) is still sufficient for our purposes. Developing a more suitable translation from natural language to logic would certainly be interesting (cf. [10]) but is beyond the scope of our project.

The subformula affected by the negation obviously includes the predicates at, math, theme and good. It is easy to see that this scope is unnecessarily large, since the word "math" is clearly not negated in the sentence. Actually, it would be sufficient to only negate the word "good". Nevertheless, "not good" may have a different meaning than "bad" for humans. This could be investigated by direct experiments with humans, but this again is beyond the scope of our project. The big negation scope is not only unpleasant from a theoretical point of view, but leads to major problems in the CoRg system when the generated formula is fed into the automated reasoning system Hyper.

Before we take a closer look at these problems, let us assume that the selected background knowledge contains the information that math is a school subject. In fact, ConceptNet contains the triple (Math,IsA, school subject) which can be translated into:

$$
\forall X(\text { math }(X) \rightarrow \text { school_subject }(X))
$$

In the CoRg system, this background knowledge together with Formula (1) is passed to the Hyper theorem prover. In a preprocessing step, Hyper converts all input formulae to 
clause normal form. For Formulae (1) and (2), this includes the following clauses:

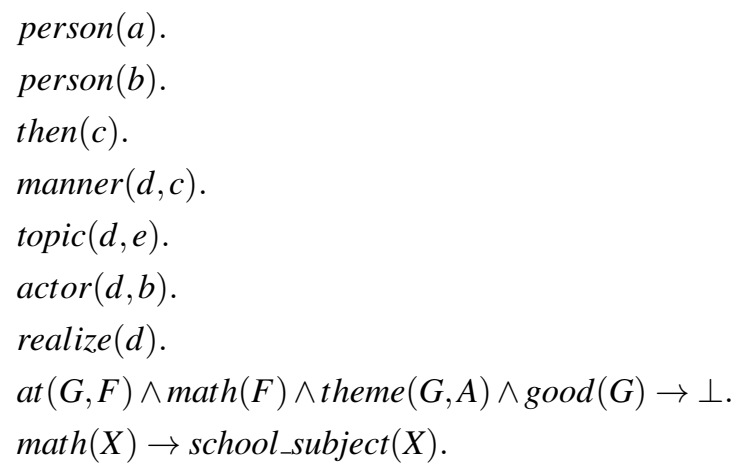

Since Hyper is based on the hypertableau calculus [8], it can use Formula (3) only to close branches. More precisely, forward reasoning performed by Hyper is quite capable of using negative knowledge during the reasoning process, but the inferred knowledge always contains only positive statements. This means that the predicate name math can never appear in an open branch of a tableau constructed by Hyper. Moreover, Formula (4), created from the background knowledge, can not be used to infer that the person in question is not good at a school subject. Therefore, the predicate names math and school_subject will not appear in (potential partial) models or inferences of Hyper. As we have seen above, the machine learning component of the CoRg system uses predicate names that occur in the models and inferences of Hyper to decide which of the given alternatives provides the right answer. Therefore, for us it is important that the output of Hyper contains as much knowledge as possible from the natural language input together with inferred knowledge from background knowledge.

In summary, the unnecessarily large scope of the negation in Formula (1) results in the fact that important information is withheld from the machine learning component of the CoRg system. Not all types of negation lead to this problem and need to be addressed by our approach: KnEWS is able to handle lexical and morphological negations, as they are directly translated into a single predicate without logical negation. Therefore, our approach only needs to address syntactic negation like "not good". But syntactic negation is common in natural language: 127 of the first 310 examples of the StoryClozeTest [30] contain syntactic negation.

Since the way KnEWS handles syntactic negation leads to the above mentioned problems in cognitive reasoning, we aim at determining the negatus for a given negation and to reduce the scope of the negation in the formula to include only the negatus. In the example, the subformula $\operatorname{good}(G)$ represents the desired negatus "good". The reduction of the scope of the negation in Formula (1) to the negatus leads to the following formula:

$$
\begin{aligned}
\exists A, B, C, D, E( & \operatorname{person}(A) \wedge \operatorname{person}(B) \wedge \operatorname{then}(C) \wedge \text { manner }(D, C) \wedge \\
& \operatorname{topic}(D, E) \wedge \operatorname{actor}(D, B) \wedge \operatorname{realize}(D) \wedge \\
& \exists F, G(\operatorname{at}(G, F) \wedge \operatorname{math}(F) \wedge \operatorname{theme}(G, A) \wedge \neg \operatorname{good}(G)))
\end{aligned}
$$


We are aware that moving the negation in the formula to the predicate representing the negatus does not preserve logical equivalence. But in our practical application context of commonsense reasoning, this is not mandatory. In addition, ordinary equivalence transformations such as De Morgan's rule do not help to restrict the scope of the negation in the formula to the negatus. But after reducing the scope of the negation of the formula to the negatus, in many cases it is even possible to remove the negation completely by determining an inverse (antonym) for the predicate symbol of the negated subformula. The negated subformula can be replaced by a non-negated subformula with the inverse as predicate symbol. For the example above, we determine the inverse bad of the predicate symbol good of the negated subformula $\neg \operatorname{good}(G)$ with the help of WordNet and substitute the negated subformula $\neg \operatorname{good}(G)$ in Formula (5) by $\operatorname{bad}(G)$.

Clausification of the resulting formula leads to a set of clauses consisting only of unit clauses. Hence Hyper can use the background knowledge and infer that math is a school_subject. All this information will become available to the machine learning component of the CoRg system. Of course, one could argue that "being not good at math" is not the same as "being bad at math", but since our goal is to allow the theorem prover to draw as many inferences as possible, we accept this imprecision and consider the replacement by an antonym as an approximation.

\subsection{Approach to Negation Treatment for Cognitive Reasoning}

Now, after having described our motivation for the determination of the exact negatus, we describe the implementation and evaluation of this approach. For a given text $T$ and its corresponding first-order logic representation $F$, Algorithm 1 describes how we treat negations. In the beginning, some preprocessing is performed (Lines 1 and 2): For the formula $F$, this means the removal of double negations. For the text $T$, this step includes lemmatization, tokenization, part-of-speech (POS) tagging and removal of certain stopwords like "the" or "he". The stopwords we use are based on the stopwords used in the Natural Language Toolkit (NLTK) Python library for Natural Language Processing. ${ }^{3}$ Since modal and auxiliary verbs and negation cues are important for our purposes, we do not take them as stopwords. Figure 3 shows the complete set of used stopwords.

Then (in Lines 3 to 10) the negation cues $x_{1}, \ldots, x_{n}$ occurring in the text $T$ as well as the logical negations $y_{1}, \ldots, y_{m}$ in the formula $F$ are considered. For each negation cue $x_{i}$, the set $M\left(x_{i}\right)$ of all words in a word window of size $k$ around (usually after) the negation cue are determined. In our evaluation we use $k=3$ which has been established by manually determining the position of the negatus relative to the position of the negation cue from 486 example negations of the CD-SCO train dataset (cf. Figure 4 for details). Other choices are possible and will be considered in future work. Depending on the strategy we consider the word preceding the negation cue to the word window. Similarly, for each logical negation $y_{j}$, the set $M\left(y_{j}\right)$ of predicates occurring in the scope of negation $y_{j}$ is determined.

\footnotetext{
${ }^{3}$ Available at: http://www.nltk.org/
} 


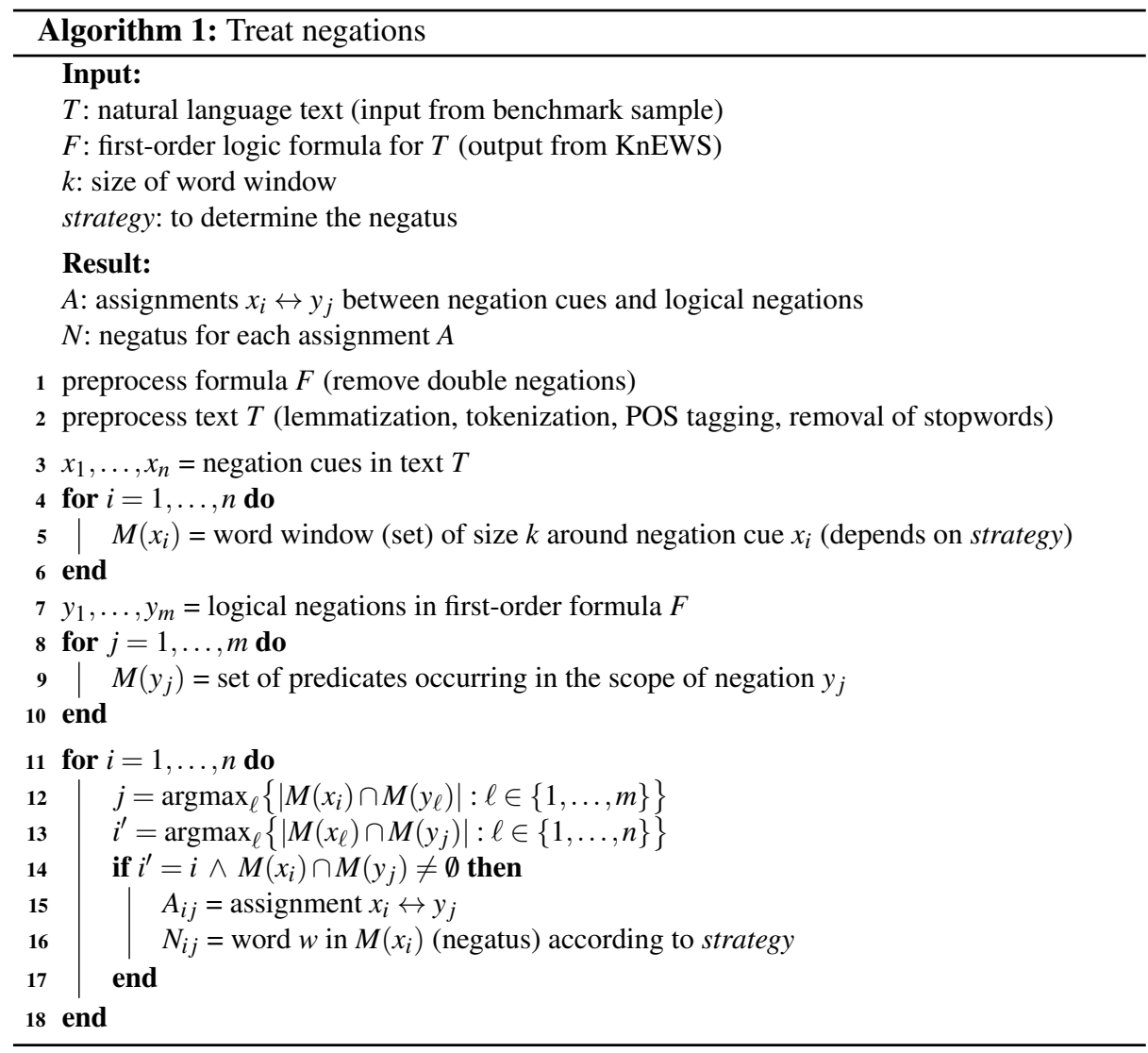

a, about, above, after, again, against, ain, all, am, an, and, any, are, as, at, because, been, before, being, below, between, both, but, by, d, did, do, does, doing, down, during, each, few, for, from, further, having, he, her, here, hers, herself, him, himself, his, how, i, if, in, into, it, it's, its, itself, just, ll, m, ma, me, more, most, my, myself, now, o, of, off, on, once, only, or, other, our, ours, ourselves, out, over, own, re, s, same, shan, she, she's, should've, so, some, such, than, that, that'll, the, their, theirs, them, themselves, then, there, these, they, this, those, through, to, too, under, until, up, ve, very, we, what, when, where, which, while, who, whom, why, will, with, won, y, you, you'd, you'll, you're, you've, your, yours, yourself, yourselves

Figure 3. Complete set of used stopwords. Note that all words in this list are lower case. So the word "i" in this list corresponds to "I". Furthermore, single letters (like "s") in this list are included in the stopwords since they can be used to remove parts of speech resulting from tokenization. For instance, "he's" is tokenized into "he" and "s". 


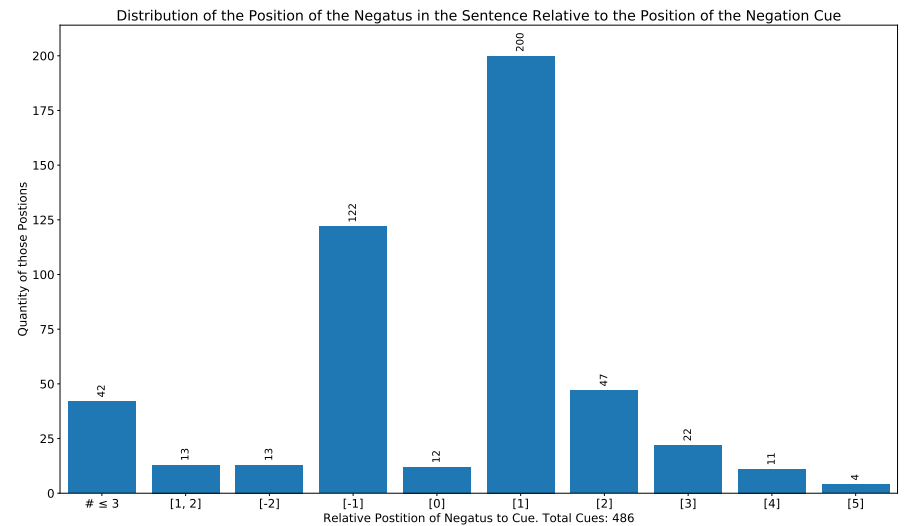

Figure 4. Distribution of the relative position of the negatus in the sentence. Positive position values indicate that the negatus occurred after the negation cue, negative values that it occurred before the cue in the sentence. For instance, in 200 cases the negatus was the word immediately following the negation cue (indicated by [1]) and in 122 cases the negatus was the word immediately preceding the cue. The position cue $[1,2]$ in the $x$-axis denotes a negatus consisting of two words, the first in position 1 and the second in position 2 after the negation cue. The bar annotated with \# $\leq 3$ comprises negatus positions which have less or equal 3 occurrences.

Texts may contain several negations. In general, the number $n$ of negation cues in the text does not coincide with the number $m$ of logical negations in the formula. Therefore, it can happen that for some negation in the text no corresponding negation can be found in the formula or vice versa. Since in addition the order of the information in the text does not always coincide with the order of the information in the generated formula, the first negation in the text does not necessarily correspond to the first negation in the formula. Hence, it is necessary to determine a mapping between both kinds of negation. We do this as follows: The negation cue $x_{i}$ and the logical negation $y_{j}$ are assigned to each other if the intersection of $M\left(x_{i}\right)$ and $M\left(y_{j}\right)$ is non-empty and has maximal cardinality with respect to both indexes $i$ and $j$ (cf. Lines 11 to 18).

Finally, for each assignment $x_{i} \leftrightarrow y_{j}$, the negatus $N_{i j}$ is determined. Which word is selected as negatus, depends on the specified strategy and thus on the word types of the words in the word window, including possibly the negation cue. The strategies considered are:

- baseline: For a negation cue that is a negated modal verb ("can't", "couldn't", "should not"), the modal verb is chosen as the negatus (i.e., "can", "could", "should"). For all other cases, the negatus is the first non-stopword in the word window. The baseline strategy was determined after manually examining the position of the negatus relative to the position of the negation cue (cf. Figure 4).

- first non-stopword strategy (FNS): Negatus is the first non-stopword in the word window. 
- first verb strategy $(F V)$ : Negatus is the first verb in the word window.

- first verb or non-stopword strategy $(F N S+F V)$ : Negatus is the first verb in the word window. Use first non-stopword strategy if no negatus was determined this way.

- combination strategy (Comb): Determine negatus word according to Table 1.

All strategies use a word window consisting of the first $k$ non-stopwords following the negation cue - with one exception: The combination strategy uses a word window of size $k$ which starts from the first non-stopword left of the negation cue. The negation cue is not included in the word window. Afterwards, if the determined negatus corresponds to a predicate name in the scope of the negation, formula $F$ is adapted such that the scope only includes this predicate. Further, if possible, the negatus predicate is replaced by its inverse, i.e., an antonym, such that eventually the negation is completely removed. Antonyms are looked up in WordNet.

Table 1. Procedure to determine the negatus in the combination strategy for syntactic negation. For each negation cue, the table is scanned from top to bottom and the first row matching the cue is selected and applied.

\begin{tabular}{ll}
\hline Negation Cue & Negatus \\
\hline $\begin{array}{l}\text { can't, cannot, can not } \\
\text { couldn't, could not } \\
\text { shouldn't, should not }\end{array}$ & $\begin{array}{l}\text { can } \\
\text { could } \\
\text { should }\end{array}$ \\
nothing, isn't, is not, aren't, are not, wasn't, was not, & \\
weren't, were not & first non-stopword in word window \\
no & first noun after negation \\
not, never, all other negation cues & first verb in word window \\
any cues, if no negatus has been determined yet & first non-stopword in word window \\
\hline
\end{tabular}

\subsection{Example for Algorithm 1}

To further illustrate this procedure, we consider the following example:

"I don't like the cookies with raisins but I can't eat the ones with chocolate either."

For convenience, the important parts are colored. The corresponding formula is:

$$
\begin{array}{r}
\exists A, B, C, D(\text { one }(A) \wedge \operatorname{person}(C) \wedge \operatorname{cookie}(B) \wedge \operatorname{person}(D) \wedge \\
\neg \exists E, F, G, H, I(\text { either }(G) \wedge \operatorname{manner}(I, G) \wedge \text { with }(I, H) \wedge \operatorname{chocolate}(H) \wedge \\
\text { theme }(I, A) \wedge \text { actor }(I, C) \wedge \text { eat }(I) \wedge \operatorname{topic}(F, E) \wedge \operatorname{actor}(F, C) \wedge \operatorname{can}(F) \wedge \\
\neg \exists J, K(\text { theme }(J, B) \wedge \operatorname{actor}(J, D) \wedge \operatorname{like}(J) \wedge \operatorname{with}(B, K) \wedge \operatorname{raisin}(K))))
\end{array}
$$


The cues and word windows are as follows, with $x_{i}$ denoting the words of the natural language representation and $y_{i}$ denoting the parts of the formula:

$$
\begin{array}{ll}
x_{1}=\text { don't } & M\left(x_{1}\right)=\{\text { like, cookies, raisins }\} \\
x_{2}=\text { can't } & M\left(x_{2}\right)=\{\text { eat, ones, chocolate }\} \\
y_{1}=\text { not } & M\left(y_{1}\right)=\{\text { either, with, chocolate, eat, can }\} \\
y_{2}=\text { not } & M\left(y_{2}\right)=\{\text { like, raisin }\}
\end{array}
$$

One can see that the negations are in reverse order here. Calculating the cardinality of the intersections for every combination of $M$ (after reducing all words to their base form, e.g., "raisins" to "raisin") leads to the following:

$$
\begin{array}{ll}
\left|M\left(x_{1}\right) \cap M\left(y_{1}\right)\right|=0 & \left|M\left(x_{2}\right) \cap M\left(y_{1}\right)\right|=2 \\
\left|M\left(x_{1}\right) \cap M\left(y_{2}\right)\right|=2 & \left|M\left(x_{2}\right) \cap M\left(y_{2}\right)\right|=0
\end{array}
$$

This leads to the assignments $x_{1} \leftrightarrow y_{2}$ as well as $x_{2} \leftrightarrow y_{1}$. To determine the negatus, we apply, e.g., the FNS-Strategy to the natural language text, which results in negatus $_{1}=$ "like" and negatus ${ }_{2}=$ "can". Their respective inverse words, which can be used to replace the negated predicates in the formula, are inverse $e_{1}=$ "dislike" and inverse $_{2}=$ "unable".

\section{Experiments}

To evaluate our approach, we use the *SEM 2012 Negation Task [28,29] as benchmark, where negations in some books by Conan Doyle are annotated (cf. Section 2.2), more precisely $\mathrm{CD}-\mathrm{SCO}$, the corpus with scope annotation that additionally provides a negatus. The sentences of the corpus are split into words which are POS-tagged. Furthermore the stem of the word and a syntax-tree for the sentence is given.

However, the *SEM 2012 Negation Task does not entirely fit to our problem. Since we only want to address syntactic negation (cf. Section 3.2), we manually created a filtered version of CD-SCO: Lexical and morphological negations are deleted, while the syntactic ones remain. This process resulted in 132 deleted samples in the train dataset, 32 deleted samples in the development (dev) dataset and 32 deleted samples in the test dataset. Additionally, we manually created an own benchmark based on the StoryClozeTest [30], taking the first 100 tasks of the StoryClozeTest that contain a syntactic negation. Each StoryClozeTest task consists of six sentences. We generated one negation task for each negation we found (126) and the second and third author of this paper annotated the negatus for each negation. We denote these benchmarks as Cloze-NEG. In addition to that, we evaluated our approach within the CoRg system using all problems from the StoryClozeTest. ${ }^{4}$

Next, we present experimental results of our approach on the CD-SCO benchmarks and compare them with results achieved by different systems in the *SEM 2012 negation task on scope resolution where also the negatus has been determined. For the *SEM

\footnotetext{
${ }^{4}$ All used benchmark sets and our implementation in Python are available at http://gitlab. uni-koblenz.de/obermaie/negationcognitivereasoning.
} 
2012 Shared Task, it was possible to enter systems in two tracks: the closed and the open track. The systems in the closed track did not use any external resources. In contrast to this, the systems in the open track were allowed to use external resources as well as tools. Since our approach relies on the KnEWS system which is built on top of Boxer [11], our approach belongs to the open track and we thus compare our approach to the systems from the open track in the following. Furthermore, we use evaluation measure $B$ of the *SEM 2012 competition where precision is computed as the number of true positives divided by the total number of a systems predictions.

\subsection{Other Approaches}

We compare our approach against other approaches, in particular the five approaches submitted to the open track of the *SEM 2012 Negation Task [28,29]: UiO2, UGroningen $\mathrm{r}$, UGroningen $\mathrm{r} 1$, UCM-1 and UCM-2. UiO2 uses conditional random fields (CRFs) and support vector machines (SVMs), while the UGroningen approaches use discourse representation structures (DRSs), and the UCM approaches rely on syntax trees and rules. Let us discuss these approaches in some more detail:

$\mathrm{UiO} 2$ [22] is the only approach using machine learning. An SVM classifier detects cues, while the CRF classifiers [23] predict the scopes and events. In the open track, $\mathrm{UiO} 2$ makes use of the MaltParser [33] and its pre-trained model to obtain dependency graphs.

The UGroningen approaches [6] are probably the most similar to our approach, as they use C\&C Tools and Boxer $[11,12]$ to produce DRSs. We use the tool KnEWS, which integrates $\mathrm{C} \& \mathrm{C}$ Tools and Boxer. Another similarity is the detection of the scope, which is extracted from the tokens that occur in the scope of the negated DRS.

The UCM approaches [2,5] implement a rule-based system. They identify the cues using a list of predefined negation words. For lexical negation cues, they add a step using WordNet antonyms to make sure that it is a negated form of an existing word. Scope detection is done by using the syntax tree of the sentence. To identify the negatus they have two strategies: If it is a lexical negation they mark the word itself as negatus, if it is a syntactical negation they mark the verb as the negatus, if it is also the next word. Our approach can be seen as a combination of the UGroningen and UCM approaches.

\subsection{Data Preparation and Evaluation}

Table 2 shows the results of our approach using different strategies and results of systems in the open track of *SEM 2012 Task 1. Since we do not use machine learning in this procedure, we present our results for the training and development set as well. For the systems of *SEM 2012 Task 1, only the results on the CD-SCO test set are shown. In the CD-SCO benchmarks, a significant proportion of examples with negation have a cue and a scope in the gold standard, but no negatus. So, the train dataset contains 3,643 text passages with a total of 3,779 lines in the gold standard data. Of these lines, 983 contain a negation. However, in only 615 of these cases, a negatus is specified in the gold standard. In contrast to this, our approach often finds a negatus in these cases. For instance, consider example baskervilles07_301 from the train dataset which contains 
Table 2. Results of our approach using the strategies described in Section 3.3 on the CD-SCO dataset in the upper part of the table. The lower part of the table depicts the result of the systems in the open track of *SEM 2012 Task 1.

\begin{tabular}{|c|c|c|c|}
\hline & Train & Dev & Test \\
\hline & Prec. Rec. $\quad F_{1}$ & Prec. Rec. $\quad F_{1}$ & Prec. Rec. $\quad F_{1}$ \\
\hline Baseline & 24.5634 .3128 .63 & 26.1731 .9728 .78 & 23.1432 .3726 .99 \\
\hline FNS & 24.5634 .3128 .63 & 26.1731 .9828 .78 & 23.1432 .3726 .99 \\
\hline $\mathrm{FV}$ & 19.7520 .6520 .19 & 22.5220 .4921 .46 & 24.4224 .2824 .35 \\
\hline $\mathrm{FV}+\mathrm{FNS}$ & 22.4731 .3826 .19 & 26.8532 .7929 .52 & 23.5532 .9527 .47 \\
\hline Comb. & 35.3949 .4341 .25 & 34.9042 .6238 .38 & 34.7148 .5540 .48 \\
\hline $\mathrm{UiO} 2$ & & & 63.8257 .4060 .44 \\
\hline UGroningen $\mathrm{r} 2$ & & & 55.2265 .2959 .83 \\
\hline UGroningen r1 & & & 52.6652 .0552 .35 \\
\hline UCM-1 & & & 66.6712 .7221 .36 \\
\hline UCM-2 & & & 44.4421 .1828 .69 \\
\hline
\end{tabular}

the sentence: "I must not stop, or my brother may miss me." The gold standard does not specify a negatus for this example. The formula created by KnEWs is as follows:

$$
\begin{array}{r}
\exists A, B, C, D(\operatorname{person}(A) \wedge \operatorname{brother}(B) \wedge \operatorname{of}(B, C) \wedge \operatorname{person}(C) \wedge \operatorname{person}(D) \wedge \\
\neg \exists E, F, G(\operatorname{actor}(G, D) \wedge \operatorname{stop}(G) \wedge \operatorname{topic}(F, E) \wedge \operatorname{actor}(F, D) \wedge \operatorname{must}(F)) \vee \\
\exists H, I, J(\text { theme }(J, A) \wedge \operatorname{actor}(J, B) \wedge \operatorname{miss}(J) \wedge \operatorname{topic}(I, H) \wedge \operatorname{actor}(I, B) \wedge \operatorname{may}(I)))
\end{array}
$$

Although no negatus is specified in the gold standard for this example, we can remove the negation in the formula with our approach by replacing the predicate stop (corresponding to the negatus) by its inverse, namely the antonym proceed. This result corresponds to the sentence "I must proceed, or my brother may miss me.". Cases like this example are counted as false in the calculation of precision in Table 2. We report numbers for the CD-SCO benchmarks in Table 3 for the filtered CD-SCO benchmarks where only negations with negatus specification in the gold standard are considered. Of course, sometimes there are cases where indeed no negatus should be specified like in direct speech and elliptic utterances, where negations refer back to the previous sentence. Additionally sometimes the gold standard contain errors. One example is from the train set with a wrongly annotated negatus: "The dinner itself was neither well served nor well cooked [...]". The gold standard states "served" and "cooked" as negatus, while we are confident that the negatus in both cases should be "well".

Although the CD-SCO benchmarks do not exactly reflect what we are trying to achieve with our approach, the option combination strategy still ranks in the midfield of the systems that participated in *SEM 2012. In addition to that, we tested our approach on the 100 manually labeled StoryClozeTest tasks, the Cloze-NEG benchmarks. The results are depicted in Table 4. To check whether our approach is suitable for our purpose of identifying the negatus, the Cloze-NEG benchmarks are the most suitable ones. This is because only these benchmarks aim to determine a word in the scope of the negation 
Table 3. Results of our approach using the strategies described in Section 3.3 on the filtered CDSCO dataset. For the calculation of precision cases where our approach found a negatus but the gold standard did not specify a negatus were not included.

\begin{tabular}{|c|c|c|c|}
\hline & Train Filtered & Dev Filtered & Test Filtered \\
\hline & Prec. Rec. $F_{1}$ & Prec. Rec. $F_{1}$ & Prec. Rec. $F_{1}$ \\
\hline Baseline & 44.5643 .8344 .19 & 44.0944 .0944 .09 & 42.2541 .1041 .67 \\
\hline FNS & 44.5643 .3844 .19 & 44.0944 .0944 .09 & 42.2541 .1041 .67 \\
\hline FV & 36.7126 .1330 .53 & 42.8629 .0334 .62 & 44.2128 .7734 .85 \\
\hline $\mathrm{FV}+\mathrm{FNS}$ & 40.7940 .1240 .46 & 47.3147 .3147 .31 & 42.9641 .7842 .36 \\
\hline Comb. & 64.0262 .9663 .49 & 58.0658 .0658 .06 & 61.9760 .2761 .11 \\
\hline
\end{tabular}

that, when replaced by its antonym, leads to the removal of the negation. The results on the StoryClozeTest benchmarks show that the option combination strategy is often able to identify the negatus. Furthermore, we observe that for all considered benchmarks, the option combination strategy seems to be the most suitable for identifying the negatus.

Table 4. Results of our approach on the 100 manually labeled StoryClozeTest tasks.

\begin{tabular}{|c|c|}
\hline & Cloze-NEG \\
\hline & Prec. Rec. $F_{1}$ \\
\hline Baseline & 59.5259 .5259 .52 \\
\hline FNS & 57.8555 .5656 .68 \\
\hline FV & 54.3349 .2151 .67 \\
\hline $\mathrm{FV}+\mathrm{FNS}$ & 56.3556 .3556 .35 \\
\hline Comb. & 66.6766 .6766 .67 \\
\hline
\end{tabular}

As explained above, an unnecessarily large scope of a negation in a formula belonging to a commonsense reasoning problem causes the Hyper reasoner in the CoRg system to perform only few inferences and, in the extreme case, to return an empty model. To evaluate the benefit of our approach within the CoRg system, we integrated our approach into the system and used it on the whole set of problems in the StoryClozeTest. By this, the amount of completely empty models was reduced by $71.95 \%$ from 164 to 46 (cf. Figure 5). Overall, the negation treatment resulted in an increase in the average number of distinct predicate names per model from 18.83 to 19.35 with an overall gain of distinct predicate names of 5,726 in 11,119 models. This demonstrates that our negation handling enables the theorem prover to derive additional information, which in return can be used by further processing, in our case the neural network. We hope that these encouraging results will facilitate using automated reasoning in commonsense reasoning. 


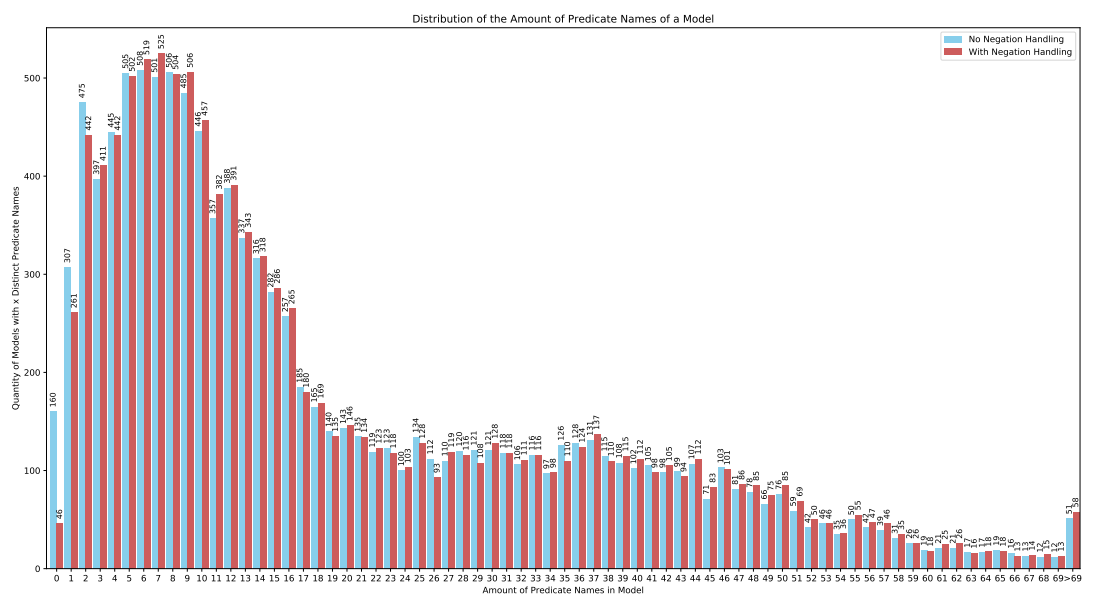

Figure 5. Distribution of the number of distinct predicate names in a model generated by Hyper with and without negation handling. WordNet was added as background knowledge. Models with more than 69 elements are comprised in one entry to enhance readability. The empty models where reduced from 164 to 46 , which is an decrease of $71.95 \%$. The average number of distinct predicates per model increased from 18.83 to 19.35 , while the absolute amount of distinct predicates increased from 209,374 to 215,100 with 5,726 new distinct predicates. Overall 11,119 models where examined. One can see that not only the elimination of empty models lead to the increase, but other models benefit from our procedure as well. For instance, the amount of models containing only one predicate decreased from 307 to 261 .

\section{Summary, Conclusions, and Future Work}

In this paper, we presented a way to detect syntactic negation in natural language using KnEWS, which is important in cognitive reasoning systems. This is why we want to replace negative information through a positive counterpart, its inverse. To achieve this, the negatus has to be identified and later on be replaced by its inverse. Our approach detects syntactic negation and identifies the negatus by combining the information from the generated KnEWS formula and from the natural language input itself.

We apply different strategies for identifying the negatus. The combination strategy apparently works best and ranks in the midfield of the *SEM 2012 negation task. Our evaluation of the StoryClozeTest yields comparable results. With the help of our approach for negation handling, we improved the input of the theorem prover Hyper in the CoRg system. This resulted in a decrease of the number of empty models by $71.95 \%$ and an average increase of 0.53 distinct predicates per model. This demonstrates an information gain which is useful for later processing in the CoRg system.

In future work, we aim to improve our negation handling by applying other strategies in more detail. Furthermore we shall use the inverse of the negatus in commonsense reasoning. So far, we implemented a first approach looking up the antonyms of 
the detected negatus from WordNet. Since eventually we work with neural networks and word embeddings (cf. [36]), another idea is to directly generate word embeddings by using an inverse operator. The thus determined antonym does not necessarily need a natural language counterpart, but would be used as an artificial word. The overall goal is to implement a cognitive reasoning system with an adequate treatment of syntactic negation.

\section{References}

1. Agirre, E., Bos, J., Diab, M., Manandhar, S., Marton, Y., Yuret, D. (eds.): *SEM 2012: The First Joint Conference on Lexical and Computational Semantics - Volume 1: Proceedings of the main conference and the shared task, and Volume 2: Proceedings of the Sixth International Workshop on Semantic Evaluation (SemEval 2012). Association for Computational Linguistics, Montréal, Canada (2012), http://www.aclweb.org/anthology/volumes/S12-1/

2. de Albornoz, J.C., Plaza, L., Díaz, A., Ballesteros, M.: UCM-1: A rule-based syntactic approach for resolving the scope of negation. In: Agirre et al. [1], pp. 282-287, http: //www.aclweb.org/anthology/S12-1037

3. Álvez, J., Lucio, P., Rigau, G.: Adimen-SUMO: Reengineering an ontology for first-order reasoning. Int. J. Semantic Web Inf. Syst. 8(4), 80-116 (2012), http://doi.org/10.4018/jswis. 2012100105

4. Antoniou, G.: A tutorial on default logics. ACM Computing Surveys 31(4), 337-359 (1999), http://doi.org/10.1145/344588.344602

5. Ballesteros, M., Díaz, A., Francisco, V., Gervás, P., De Albornoz, J.C., Plaza, L.: UCM-2: a rule-based approach to infer the scope of negation via dependency parsing. In: Agirre et al. [1], pp. 288-293, http://www.aclweb.org/anthology/S12-1038.pdf

6. Basile, V., Bos, J., Evang, K., Venhuizen, N.: UGroningen: Negation detection with discourse representation structures. In: Agirre et al. [1], pp. 301-309, http://www.aclweb.org/ anthology/S12-1040

7. Basile, V., Cabrio, E., Schon, C.: KNEWS: Using logical and lexical semantics to extract knowledge from natural language. In: Proceedings of the European Conference on Artificial Intelligence (ECAI) (2016), http://hal.inria.fr/hal-01389390

8. Baumgartner, P., Furbach, U., Pelzer, B.: The hyper tableaux calculus with equality and an application to finite model computation. Journal of Logic and Computation 20(1), 77-109 (2010), http://doi.org/10.1093/logcom/exn061

9. Bender, M., Pelzer, B., Schon, C.: System description: E-KRHyper 1.4 - extensions for unique names and description logic. In: Bonacina, M.P. (ed.) Automated Deduction - CADE 24. pp. 126-134. LNCS 7898, Springer (2013), http://doi.org/10.1007/ 978-3-642-38574-2_8

10. Blackburn, P., Bos, J., Kohlhase, M., de Nivelle, H.: Inference and computational semantics. In: Bunt, H., R., M., Thijsse (eds.) Computing Meaning. pp. 11-28. No. 77 in Studies in Linguistics and Philosophy, Springer, Dordrecht (2001), http://link.springer.com/chapter/10. 1007/978-94-010-0572-2_2

11. Bos, J.: Wide-coverage semantic analysis with Boxer. In: Semantics in Text Processing. STEP 2008 Conference Proceedings. pp. 277-286. College Publications (2008), http://www. aclweb.org/anthology/W08-2222

12. Curran, J.R., Clark, S., Bos, J.: Linguistically motivated large-scale NLP with C\&C and Boxer. In: Proceedings of the ACL 2007 Demo and Poster Sessions. pp. 33-36. Prague, Czech Republic (2007), http://aclanthology.org/P07-2009/ 
13. Devlin, J., Chang, M., Lee, K., Toutanova, K.: BERT: pre-training of deep bidirectional transformers for language understanding. In: Burstein, J., Doran, C., Solorio, T. (eds.) Proceedings of the 2019 Conference of the North American Chapter of the Association for Computational Linguistics: Human Language Technologies, NAACL-HLT, Volume 1 (Long and Short Papers). pp. 4171-4186. Association for Computational Linguistics (2019), http://aclweb.org/anthology/papers/N/N19/N19-1423/

14. Furbach, U., Hölldobler, S., Ragni, M., Schon, C., Stolzenburg, F.: Cognitive reasoning: A personal view. KI 33(3), 209-217 (2019), http://link.springer.com/article/10.1007/ s13218-019-00603-3

15. Habernal, I., Wachsmuth, H., Gurevych, I., Stein, B.: SemEval-2018 task 12: The argument reasoning comprehension task. In: Proceedings of The 12th International Workshop on Semantic Evaluation. pp. 763-772. Association for Computational Linguistics, New Orleans, Louisiana (Jun 2018), http://www.aclweb.org/anthology/S18-1121

16. Horn, L.R., Wansing, H.: Negation. In: Zalta, E.N. (ed.) Stanford Encyclopedia of Philosophy. Metaphysics Research Lab, Stanford University (2020), http://plato.stanford.edu/ entries/negation/

17. Janssen, T.M.V., Zimmermann, T.E.: Montague semantics. In: Zalta, E.N. (ed.) Stanford Encyclopedia of Philosophy. Metaphysics Research Lab, Stanford University (2021), http: //plato.stanford.edu/archives/sum2021/entries/montague-semantics/

18. Jiménez-Zafra, S.M., Morante, R., Teresa Martín-Valdivia, M., Ureña-López, L.A.: Corpora annotated with negation: An overview. Computational Linguistics 46(1), 1-52 (2020), http: //doi.org/10.1162/coli_a_00371

19. Jurafsky, D., H. James, M.: Vector semantics and embeddings. In: Speech and Language Processing: an Introduction to Natural Language Processing, Computational Linguistics, and Speech Recognition, chap. 6, pp. 96-126. Prentice Hall, Upper Saddle River, N.J, 3rd edn. (2020), http://web.stanford.edu/ jurafsky/slp3/ed3book.pdf, draft

20. Kamp, H., Reyle, U.: From Discourse to Logic: An Introduction to Modeltheoretic Semantics of Natural Language, Formal Logic and Discourse Representation Theory. Springer, Dordrecht (1993), http://www.springer.com/de/book/9780792310280

21. Kolhatkar, V., Wu, H., Cavasso, L., Francis, E., Shukla, K., Taboada, M.: The SFU opinion and comments corpus: A corpus for the analysis of online news comments. Corpus Pragmatics 4, 155-190 (2020), http://link.springer.com/article/10.1007/s41701-019-00065-w

22. Lapponi, E., Velldal, E., Øvrelid, L., Read, J.: UiO 2: Sequence-labeling negation using dependency features. In: Agirre et al. [1], pp. 319-327, http://www.aclweb.org/anthology/ S12-1042

23. Lavergne, T., Cappé, O., Yvon, F.: Practical very large scale CRFs. In: Proceedings of the 48th Annual Meeting of the Association for Computational Linguistics. pp. 504-513 (2010), http://www.aclweb.org/anthology/P10-1052

24. Liu, H., Singh, P.: ConceptNet - a practical commonsense reasoning tool-kit. BT Technology Journal 22(4), 211-226 (2004), http://doi.org/10.1023/B:BTTJ.0000047600.45421.6d

25. Lyons, J.: Semantics, vol. 2. Cambridge University Press, Cambridge, New York, Melbourne, Madrid (1977), http://doi.org/10.1017/CBO9780511620614

26. Maslan, N., Roemmele, M., Gordon, A.S.: One hundred challenge problems for logical formalizations of commonsense psychology. In: Twelfth International Symposium on Logical Formalizations of Commonsense Reasoning, Stanford, CA (2015), http://www.aaai.org/ocs/ index.php/SSS/SSS15/paper/viewFile/10252/10080

27. Miller, G.A.: WordNet: a lexical database for english. Commun. ACM 38(11), 39-41 (1995), http://doi.org/10.1145/219717.219748

28. Morante, R., Blanco, E.: *SEM 2012 shared task: Resolving the scope and focus of negation. In: Agirre et al. [1], pp. 265-274, http://www.aclweb.org/anthology/S12-1035 
29. Morante, R., Daelemans, W.: ConanDoyle-neg: Annotation of negation cues and their scope in conan doyle stories. In: Proceedings of the Eighth International Conference on Language Resources and Evaluation (LREC'12). pp. 1563-1568. European Language Resources Association (ELRA), Istanbul, Turkey (2012), http://www.lrec-conf.org/proceedings/lrec2012/ pdf/221_Paper.pdf

30. Mostafazadeh, N., Roth, M., Louis, A., Chambers, N., Allen, J.: LSDSem 2017 shared task: The story cloze test. In: Proceedings of the 2nd Workshop on Linking Models of Lexical, Sentential and Discourse-level Semantics. pp. 46-51 (2017), http://doi.org/10.18653/ v1/w17-0906

31. Mueller, E.T.: Commonsense Reasoning. Morgan Kaufmann, San Francisco, 2nd edn. (2014), http://dl.acm.org/doi/book/10.5555/2821577

32. Niven, T., Kao, H.Y.: Probing neural network comprehension of natural language arguments. In: Proceedings of the 57th Annual Meeting of the Association for Computational Linguistics. pp. 4658-4664. Association for Computational Linguistics, Florence, Italy (Jul 2019), http://www.aclweb.org/anthology/P19-1459

33. Nivre, J., Hall, J., Nilsson, J.: MaltParser: A data-driven parser-generator for dependency parsing. In: LREC. vol. 6, pp. 2216-2219 (2006), http://aclanthology.org/L06-1084/

34. Radford, A., Narasimhan, K., Salimans, T., Sutskever, I.: Improving language understanding by generative pre-training. Tech. rep., Open AI (2018), http://openai.com/blog/ language-unsupervised/

35. Schon, C., Siebert, S., Stolzenburg, F.: The CoRg project: Cognitive reasoning. KI 33(3), 293-299 (2019), http://link.springer.com/article/10.1007/s13218-019-00601-5

36. Siebert, S., Schon, C., Stolzenburg, F.: Commonsense reasoning using theorem proving and machine learning. In: Holzinger, A., Kieseberg, P., Tjoa, A.M., Weippl, E. (eds.) Machine Learning and Knowledge Extraction - Proceedings of CD-MAKE 2019. pp. 395-413. LNCS 11713, Springer Nature Switzerland, Canterbury, UK (2019), http://doi.org/10.1007/ 978-3-030-29726-8_25

37. Simari, G.R., Loui, R.P.: A mathematical treatment of defeasible reasoning and its implementation. Artificial Intelligence 53(2-3), 125-157 (1992), http://doi.org/10.1016/0004-3702(92) 90069-A

38. Speer, R., Chin, J., Havasi, C.: ConceptNet 5.5: An open multilingual graph of general knowledge. In: AAAI Conference on Artificial Intelligence. pp. 4444-4451 (2017), http: //aaai.org/ocs/index.php/AAAI/AAAI17/paper/view/14972

39. Wirth, C.P., Stolzenburg, F.: A series of revisions of David Poole's specificity. Annals of Mathematics and Artificial Intelligence 78(3), 205-258 (2016), http://doi.org/10.1007/ s10472-015-9471-9, special issue on Belief Change and Argumentation in Multi-Agent Scenarios. Issue editors: Jürgen Dix, Sven Ove Hansson, Gabriele Kern-Isberner, Guillermo Simari 